\title{
Range resolution improvement in passive bistatic radars using nested FM channels and least squares approach
}

Musa Tunç Arslan

Mohammad Tofighi

Rasim Akın Sevimli

Ahmet Enis Çetin 


\title{
Range Resolution Improvement in Passive Bistatic Radars using Nested FM Channels and Least Squares Approach
}

\author{
Musa Tunç Arslan, Muhammad Tofighi, Rasim Akın Sevimli, Ahmet Enis Çetin * \\ Bilkent University, Dept. of Electrical and Electronics Engineering, Ankara, Turkey;
}

\begin{abstract}
One of the main disadvantages of using commercial broadcasts in a Passive Bistatic Radar (PBR) system is the range resolution. Using multiple broadcast channels to improve the radar performance is offered as a solution to this problem. However, it suffers from detection performance due to the side-lobes that matched filter creates for using multiple channels. In this article, we introduce a deconvolution algorithm to suppress the side-lobes. The two-dimensional matched filter output of a PBR is further analyzed as a deconvolution problem. The deconvolution algorithm is based on making successive projections onto the hyperplanes representing the time delay of a target. Resulting iterative deconvolution algorithm is globally convergent because all constraint sets are closed and convex. Simulation results in an FM based PBR system are presented.
\end{abstract}

Keywords: passive bistatic radar, range resolution, deconvolution

\section{INTRODUCTION}

Passive Bistatic Radar (PBR) systems exploit an illuminator of opportunity, often commercial ones such as FM, digital audio broadcast (DAB) and digital video broadcasts (DVB). Since the transmitted waveform is commercial, it cannot be changed to improve the radar performance. One of the main drawback of a PRB system is the range resolution. Radar range resolution is inversely proportional with the bandwidth of the broadcast signal. In general, $200 \mathrm{kHz}$ bandwidth is nominated for commercial FM broadcasts which results with $1.5 \mathrm{~km}$ two sided range resolution. However, this is rarely achieved, FM broadcast signal bandwidth depends heavily on the type of broadcast at the time. A news program causes FM broadcast to have less bandwidth whereas a rock music produces large bandwidth. ${ }^{1}$

In addition to this, detection of close proximity targets is further limited by the classical method of timedelay estimation, the matched filter. Under the Gaussian white noise assumption and known number of echoes, matched filter is the optimal maximum likelihood receiver. However, the number of echoes cannot be known beforehand. With unknown number of echoes, and if the echoes are close to each other, matched filter looses its optimality. $^{2}$

A solution to range resolution problem is to use DAB and DVB as illuminators of opportunity which have a high bandwidth of, $1.5 \mathrm{MHz}$ for $\mathrm{DAB}$ and $2-8 \mathrm{MHz}$ for DVB-T, respectively. This results with better range resolution. However, these methods suffer from low range performance due to the orthogonal frequency modulation scheme they use and the transmitter characteristics. ${ }^{3}$ An FM based PBR can detect targets up to $200 \mathrm{~km}$. On the other hand a DAB based PBR system can detect targets up to $70 \mathrm{~km} .{ }^{4,5}$

In order to overcome the range resolution problem, the use of multiple FM channels is proposed. ${ }^{6,7}$ In Ref. 6 , it is shown that concatenated multiple FM channels in frequency domain improves range resolution. However, side-lobes next to target peaks are also generated at the output of the standard matched filter. Target detection algorithms such as CFAR may classify side-lobes as false targets. In Ref. 6, a frequency domain method is developed as a solution to the side-lobe problem. The frequency domain method is based on division and it requires a low-pass filter to prevent division by zero and noise amplification.

* This work was supported in part by the Scientific and Technical Research Council of Turkey, TUBITAK, under project 113A010. Any opinion, determination and conviction is not the official opinion of TUBITAK in the publication according to the contract

Further author information: (Send correspondence to M.T.A.)

M.T.A.: E-mail: mtarslan@ee.bilkent.edu.tr

Signal Processing, Sensor/Information Fusion, and Target Recognition XXIV,

edited by Ivan Kadar, Proc. of SPIE Vol. 9474, $947414 \cdot$ - 2015 SPIE

CCC code: $0277-786 \mathrm{X} / 15 / \$ 18 \cdot$ doi: $10.1117 / 12.2176067$

Proc. of SPIE Vol. 9474 947414-1 
In this paper, we propose a time domain deconvolution scheme to resolve targets that are in close proximity. Our method not only estimates the locations of targets that are in close proximity, but also reduces the side-lobes and enhances the overall range resolution performance of a multichannel PBR system. The article is organized as follows: In Section II multichannel FM based PBR systems are reviewed. In Section III the deconvolution problem and proposed deconvolution algorithm is described. In Section IV simulation results are presented.

\section{MULTICHANNEL FM BASED PBR}

In Ref. 6,7, multiple FM channels are used in matched filter to increase the total bandwidth of the input signal, which also increases the range resolution. There are two ways to produce the multi-FM channel signal for a PBR system.

In Ref. 6, a band wider than one FM channel is transferred to baseband as if it is a single FM channel and then fed to the matched filter as input. Thus multiple consecutive FM channels are present in the signal and higher range resolution is achieved. In practice, all FM channels are expected to have different content, and since the bandwidth of an FM channel heavily depends on the content, this method have some performance problems on its own.

Another method to generate multichannel FM signal is presented in Ref. 7. Different FM channels are transferred to baseband independently of each other and sampled. Sampled baseband FM signals are then modulated in frequency domain and added onto each other using digital signal processing as shown in Fig. 1. One advantage of this method is that it enables radar to use FM channels that have high bandwidth and does not suffer the problems in the first method. Since, the Doppler shift of a target is dependent on the carrier frequency of FM channels, use of FM channels anywhere from the FM spectrum is will cause Doppler ambiguity in addition to the range ambiguity. Block diagram of the multichannel FM signal generator system is in Fig. 1. In the block diagram, $s_{1}(t), s_{1}(t), \ldots, s_{M}(t)$ are the $M$ FM signals with carrier frequencies $f_{1}, f_{2}, f_{3}, \ldots, f_{M}$, respectively, $F_{s}$ is the sampling frequency of the ADC which has to satisfy the condition, $F_{s} \geq(M-1) \Delta f+200$ $\mathrm{kHz}$. In this paper, second method is used to generate the multichannel FM signal. Doppler ambiguity beyond

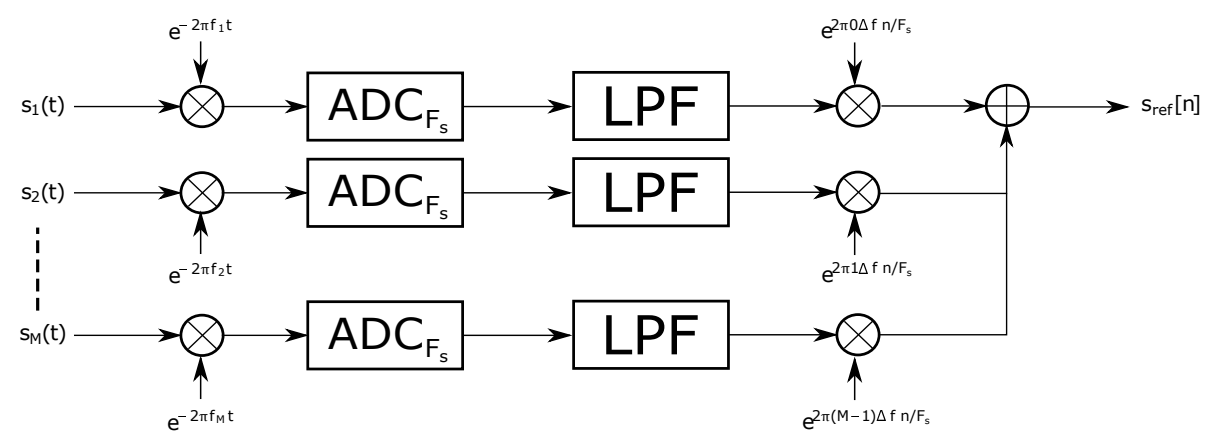

Figure 1: Block diagram of FM channel rearrangement in the PBR system.

the scope of this paper, thus it is neglected. An example of multichannel FM signal rearrangement for $M=3$ FM channels is shown in Fig. 2b and Fig. 2a for $\Delta f=200 \mathrm{kHz}$ and $\Delta f=100 \mathrm{kHz}$ respectively. Naturally, $\Delta f=100 \mathrm{kHz}$ case has less overall bandwidth because of the overlaps in the frequency domain. Thus this case has range resolution, however, matched filter output side-lobe amplitudes are also significantly less than the $\Delta f=200 \mathrm{kHz}$ case as shown in Fig. 2c and Fig. 2d respectively.

\section{PROBLEM DEFINITION}

In general, in a multichannel FM PBR system the observed signal $s_{\text {surv }}$ is of the following form:

$$
s_{\text {surv }}(t)=\sum_{p=1}^{P} a_{p} s\left(t-\tau_{p}\right) e^{j 2 \pi f_{p} t}+\nu(t)
$$


where $a_{p}$ is the attenuation of the signal, $s(t)$ is the reference signal that generated is by multiple FM channels using the block diagram shown in Fig. $1, \tau_{p}$ is the time delay of the signal echoing from the $p^{\text {th }}$ target, $f_{p}$ is the Doppler shift caused on the signal by the $p^{t h}$ target and $\nu(t)$ is the noise.

The classical method of the time of arrival estimation is the matched filter. In PBR systems the transmitted waveform is not available to the radar and thus it is acquired from a separate antenna monitoring the reference signal $s_{\text {ref }}(t)$. Signals $s_{\text {ref }}(t)$ and $s_{\text {surv }}(t)$ are sampled and discrete time versions $s_{r e f}[n]$ and $s_{\text {surv }}[n]$ are used in a matched filter. The matched filter output $\xi[l, k]$ of a PBR system for sampled signals is obtained as follows:

$$
\xi[l, k]=\sum_{n=0}^{N-1} s_{s u r v}[n] s_{r e f}^{*}[n-l] e^{j 2 \pi k n / N}
$$

where $k=-K,-K+1, \ldots,-1,0,1, \ldots, K-1, K$ are the sampled frequency bins of the matched filter, $l=$ $0,1,2, \ldots, L$ is the sampled time delay bins. Spectra of $s_{r e f}[n]$ for $\Delta f=100 \mathrm{kHz}$ and $\Delta f=200 \mathrm{kHz}$ is in Fig. $2 \mathrm{a}$ and $2 \mathrm{~b}$, respectively. Corresponding matched filter outputs are shown in Fig. 2c and 2d, respectively.

Although the matched filter output is two-dimensional we will process each row separately. Therefore we made the first variable $k$ as a subscript in Eq. (2). For each Doppler frequency index $k$ it is possible to express

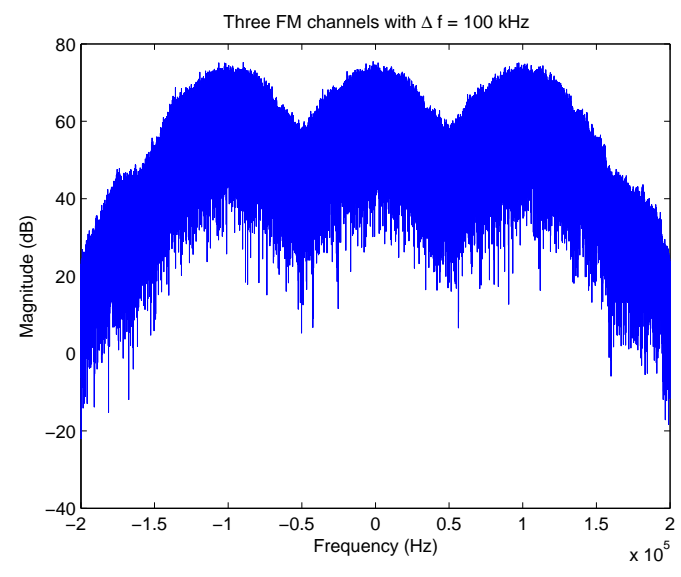

(a) spectrum of $M=3 \mathrm{FM}$ channels with $\Delta f=100 \mathrm{kHz}$.

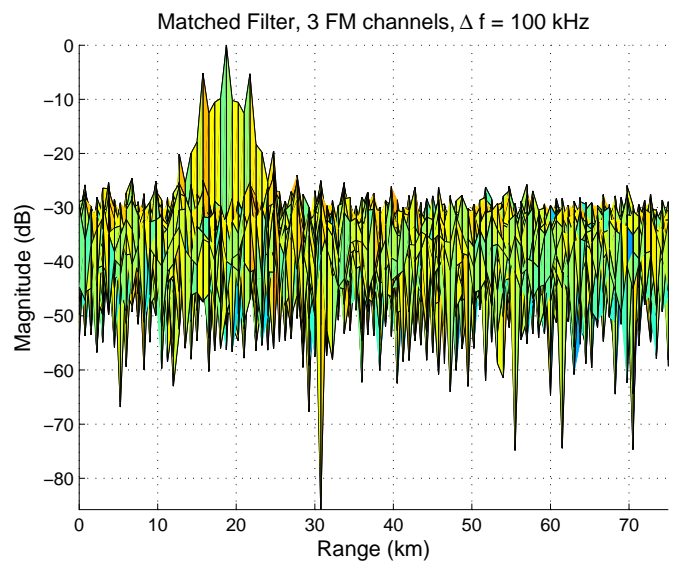

Matched filter output for 1 target, (c) $M=3 \mathrm{FM}$ channels with $\Delta f=100$ $\mathrm{kHz}$.

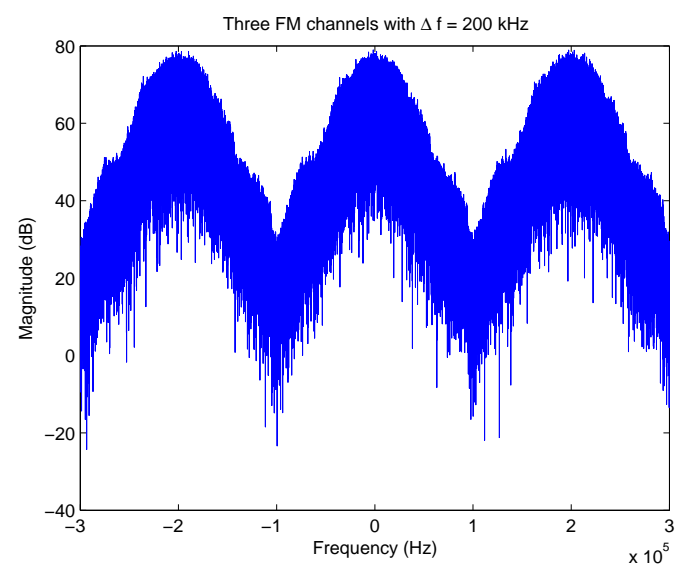

(b) spectrum of $M=3$ FM channels with $\Delta f=200 k H z$.

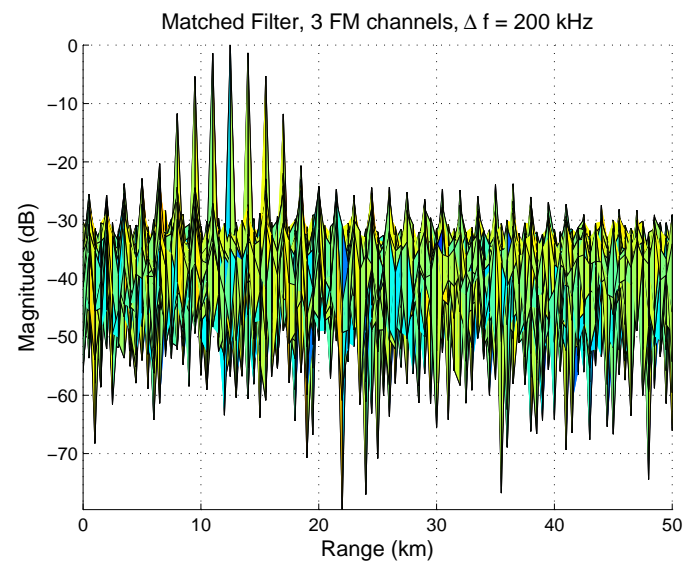

Matched filter output for 1 target,

(d) $M=3 \mathrm{FM}$ channels with $\Delta f=200$ $\mathrm{kHz}$.

Figure 2: FM channel rearrangement for different $\Delta f$ values and corresponding matched filter outputs for single target at $25 \mathrm{~km}$. 
the matched filter output,

$$
\xi_{k}[l]=\sum_{p=1}^{P} a_{p} r\left(l-l_{p}\right)+\nu[l]
$$

where $a_{p}$ is the attenuation of the $p^{t h}$ target, $r(n)$ is the auto correlation of the $s_{\text {ref }}[n]$ and the $\nu$ is the additive noise term convolved with the reference signal $s_{\text {ref }}[n] .{ }^{6}$ Eq. (3) can be expressed as:

$$
\xi_{k}[l]=(h * r)[l]+\nu[l]
$$

where

$$
h[l]=\sum_{p=1}^{P} a_{p} \delta\left[l-l_{p}\right]
$$

which is a time-invariant system representing $p$ targets with amplitudes $a_{p}$ and target delays $l_{p}$.

In ordinary PBR systems, $\xi_{k}[l]$ is computed using Eq. (2). However, it is also equivalent to Eq. (4) in which solving $h[l]$ is a deconvolution problem. The goal of PBR system is to estimate the number of targets and target delays, i.e., the system $h[l]$, which can be determined by solving a deconvolution problem. Because $\psi_{k}[l]$ is available at the output of the matched filter and $r($.$) is the autocorrelation of s_{r e f}[n]$. Therefore the system $h$ (or equivalently time delays of targets) can be estimated by solving a deconvolution problem.

\section{DECONVOLUTION USING PROJECTIONS ONTO EPIGRAPH SET OF CONVEX COST FUNCTION (PESC)}

Deconvolution problem is solved using the method of projection on to convex set (POCS) framework. ${ }^{8-15}$ The $^{\text {That }}$ following family of sets corresponding to each output value of the matched filter are considered:

$$
\mathrm{C}_{k, l}=\left\{h_{k, l} \in \mathbb{R}^{N} \mid \xi_{k}[l]=(h * r)[l]\right\} \quad l=1,2, \ldots, L .
$$

Each set $C_{k, l}$ represents the set of systems $h$ producing $\xi_{k}[l]$ at the matched filter output. The set $C_{k, l}$ correspond to a hyperplane. Therefore, sets $C_{k, l}$ are all closed and convex sets.

In POCS framework a solution $\mathbf{h}^{\star}$ which is in the intersection of sets $\mathrm{C}_{k, l}$ can be found by making successive orthogonal projections onto hyperplanes, provided that the intersection is non-empty. Iterations are stopped after projections no longer produce a significant change on interates. Projection onto the set $\mathrm{C}_{k, l}$ is computed as follows: ${ }^{16}$

$$
\mathbf{h}_{n+1}=\mathbf{h}_{n}+\frac{\xi_{k}[l]-\left(\mathbf{h}_{n} * \mathbf{r}\right)[l]}{\|\mathbf{r}\|^{2}} \mathbf{r}^{T},
$$

where $\mathbf{h}_{i}$ is the $i^{\text {th }}$ iterate which is the vector form of the systems $\mathrm{h}, \mathbf{h}_{i+1}$ is the projection vector onto the hyperplane $\mathrm{C}_{k, l}$, and $\mathbf{r}$ is the vector form of the autocorrelation $r[n]$. The iterates $\mathbf{h}_{i}$ converge to a solution $\mathbf{h}^{*}$ which is given by

$$
\lim _{i \rightarrow \infty} h_{i}=h^{*}
$$

where $h^{*} \in \underset{k, l}{\cap} C_{k, l}$ because of POCS theorem. ${ }^{8-15}$ The block diagram of the PBR system with a deconvolution unit is shown in Fig. 3.

\section{SIMULATION RESULTS}

\subsection{Example 1}

In the first simulation example, we compare the performance of standard matched filter with single channel FM signal to multichannel FM signal. For this purpose, we take the autocorrelation of the reference signal $s_{\text {ref }}[n]$. The aim of this simulation is to show the increase in range resolution $\Delta R$ for multichannel system and the effect of using different $\Delta f$ values. In this simulation, length of the autocorrelation sequence is taken as, $L=-150,-149, \ldots,-1,0,1, \ldots, 149,150$. System setup is in Table 1 . 
Table 1: System scenario for the first simulation setup.

\begin{tabular}{|c|c|c|c|}
\hline Case & \# of FM channels $(\mathrm{M})$ & $\Delta f(\mathrm{kHz})$ & Theoretical $\Delta R$ (meters) \\
\hline 1 & 1 & - & 1500 \\
\hline 2 & 3 & $100 \mathrm{kHz}$ & 750 \\
\hline 3 & 3 & $200 \mathrm{kHz}$ & 500 \\
\hline 4 & 5 & $100 \mathrm{kHz}$ & 500 \\
\hline 5 & 5 & $200 \mathrm{kHz}$ & 300 \\
\hline
\end{tabular}

Simulation results for the setup is in Fig. 5a - 5e for cases 1-5, respectively. In the simulation results, autocorrelation sequences are the same which implies that using multichannel FM signal increases the range resolution without a doubt. In addition to this, multichannel FM signal induces a side-lobe problem at the output of the matched filter. As $\Delta f$ increases, the side-lobes are stronger and closer to the main peak which may cause problems at the detection stage. Less $\Delta f$ solves lowers the overall side-lobe level at the expense of range resolution.

\subsection{Example 2}

In the first simulation, comparison of matched filter for different number of channels and different $\Delta f$ values is shown. Expected range resolution for $L=5$ channels, $\Delta f=100 \mathrm{kHz}$ is about 500 meters and $L=5$ channels, $\Delta f=200 \mathrm{kHz}$ is about 300 meters.

In this simulation, scenario in table 2 is considered. Receiver antenna is considered to be at the center of Cartesian coordinate system. In addition to this, for simplicity, transmitter is considered to be co-sited with the receiver. In modern FM transmitter systems, multiple channels broadcast from a centralized site and antenna, thus all FM channel transmitters can be considered co-sited as well.

Table 2: System scenario for the second simulation setup.

\begin{tabular}{|l|c|c|c|}
\hline & Distance to receiver (Km) & Doppler shift $(\mathrm{Hz})$ & SNR (dB) \\
\hline target $_{1}$ & 25 & 0 & $-10 \mathrm{~dB}$ \\
\hline target $_{2}$ & 26 & 0 & $-10 \mathrm{~dB}$ \\
\hline
\end{tabular}

Simulation results are in Fig. 4a and $4 \mathrm{a}$ for $\Delta f=100 \mathrm{kHz}$ and $\Delta f=200 \mathrm{kHz}$, respectively.

In the matched filter result for $\Delta f=200 \mathrm{kHz}$, side-lobes are as powerful as the main peaks. The most powerful side-lobe is $1.3 \mathrm{~dB}$ lower than the target peak. Deconvolution algorithm is able to suppress the sidelobes as expected. The two peaks corresponding to the targets are detected with a slight error in range and the most powerful side-lobe is $7 \mathrm{~dB}$ lower than the target peaks which is a considerable improvement.

For the $\Delta f=100 \mathrm{kHz}$ case, strongest side-lobe is already quite lower than the target peaks, about $4.5 \mathrm{~dB}$. Deconvolution algorithm further enhances this, suppresses the strongest side-lobe to $10 \mathrm{~dB}$ lower than the target peaks and is able to estimate the distances of targets with a slight error.

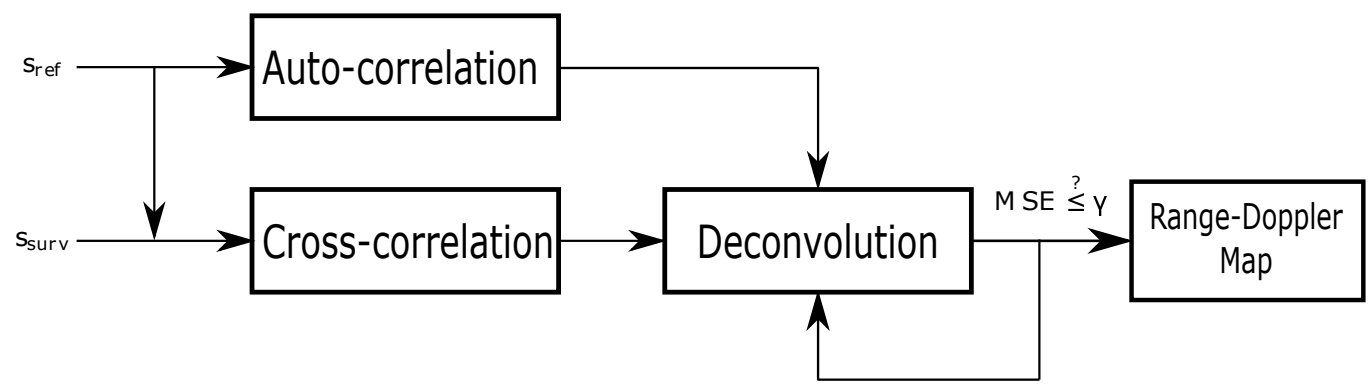

Figure 3: Deconvolution for range resolution improvement system block. 


\subsection{Example 3}

For the last simulation, we compare the single channel FM signal with matched filter to multichannel FM signal with matched filter-deconvolution combination. The simulations are conducted in a radar scenario in the Table 3. Receiver antenna is considered to be at the center of Cartesian coordinate system and transmitter is considered to be co-sited with the receiver as in the second example.

Table 3: System scenario for the third simulation setup.

\begin{tabular}{|c|c|c|c|}
\hline & Distance to receiver $(\mathrm{Km})$ & Doppler $(\mathrm{Hz})$ & SNR $(\mathrm{dB})$ \\
\hline Transmitter & $0(\mathrm{co}-$ sited $)$ & - & - \\
\hline Receiver & - & - & - \\
\hline target $_{1}$ & 20 & 25 & -10 \\
\hline target $_{2}$ & 21 & 25 & -10 \\
\hline target $_{3}$ & 60 & -30 & -10 \\
\hline target $_{4}$ & 61 & -30 & -15 \\
\hline target $_{5}$ & 62 & -30 & -20 \\
\hline
\end{tabular}

Simulation results are shown in Fig. 6a and 6b. In Fig. 6b there are only five significant peaks and all belong to the targets whereas in Fig. 6a there are more than five peaks. In addition to this, radar is able to separate targets that are only $1 \mathrm{~km}$ away from each other, which is far better than what a single channel FM signal could achieve in a typical PBR system.

\section{CONCLUSION}

In this article, we propose a multichannel FM signal based system combined with a novel deconvolution algorithm. We were able to both increase the range resolution of the PBR system and also suppress the side-lobes that are generated as a result of the matched

filter. The deconvolution algorithm is based on POCS framework and it is globally convex because all constraint sets are closed and convex. We are able to surpass the performance of other side-lobe suppression method presented in Ref. 6 in all the examples that we tried. Our deconvolution algorithm effectively increases the overall detection performance of the matched filter.
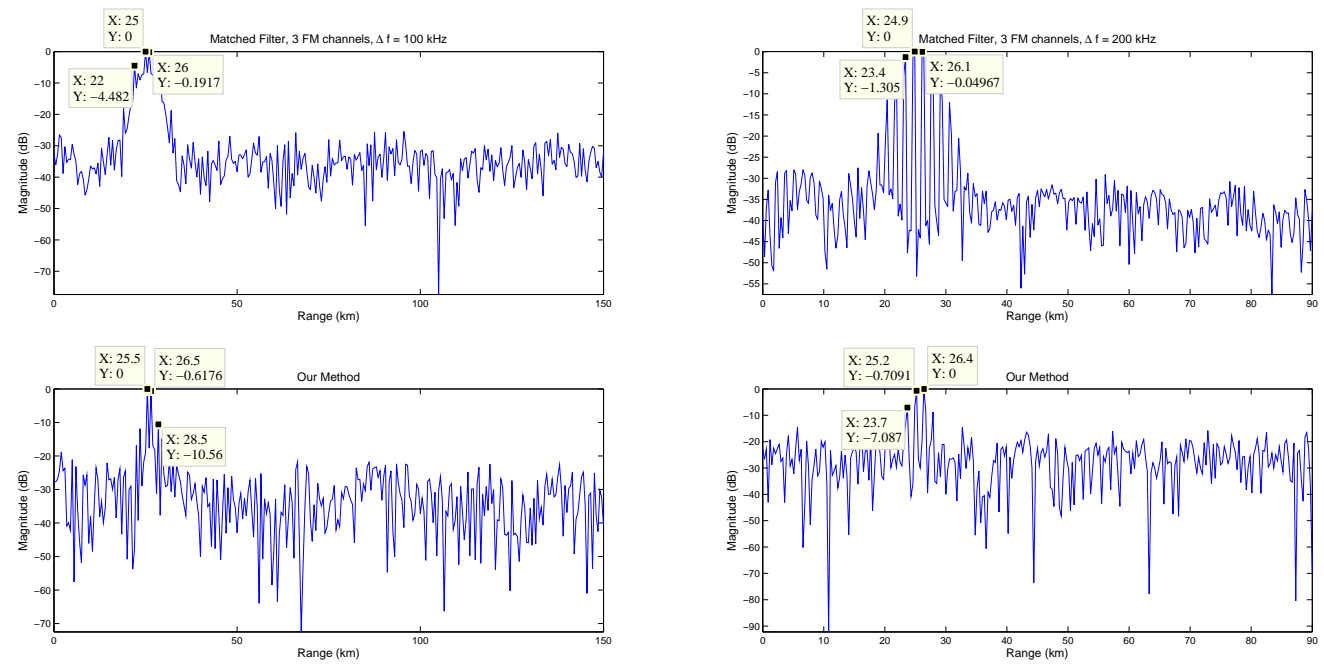

Matched filter and deconvolution results

(a) for $M=5 \mathrm{FM}$ channels with $\Delta f=$ $100 \mathrm{kHz}$ and scenario table 2 .

Matched filter and deconvolution results

(b) for $M=5 \mathrm{FM}$ channels with $\Delta f=$ $200 k \mathrm{~Hz}$ and scenario table 2 .

Figure 4: Comparison of matched filter versus deconvolution algorithm for scenario in table 2. 


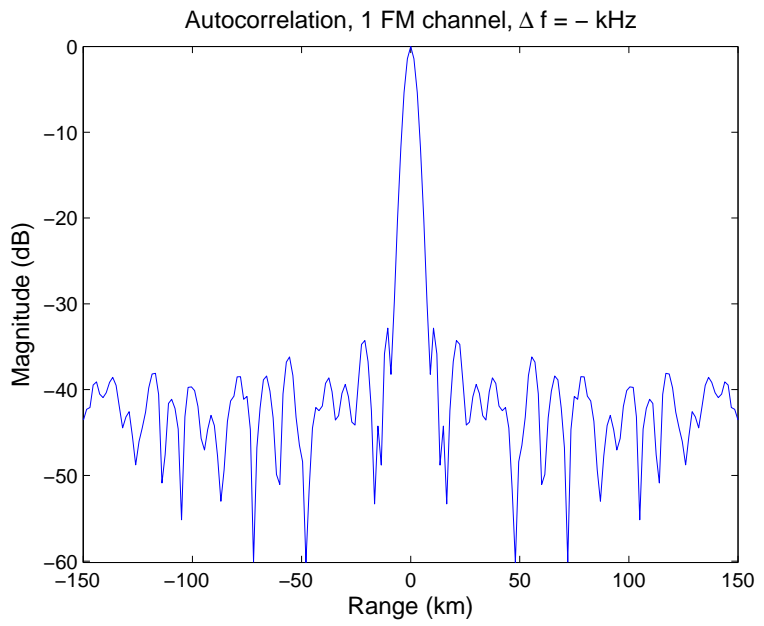

(a) Matched filter results for $M=1 \mathrm{FM}$ channels
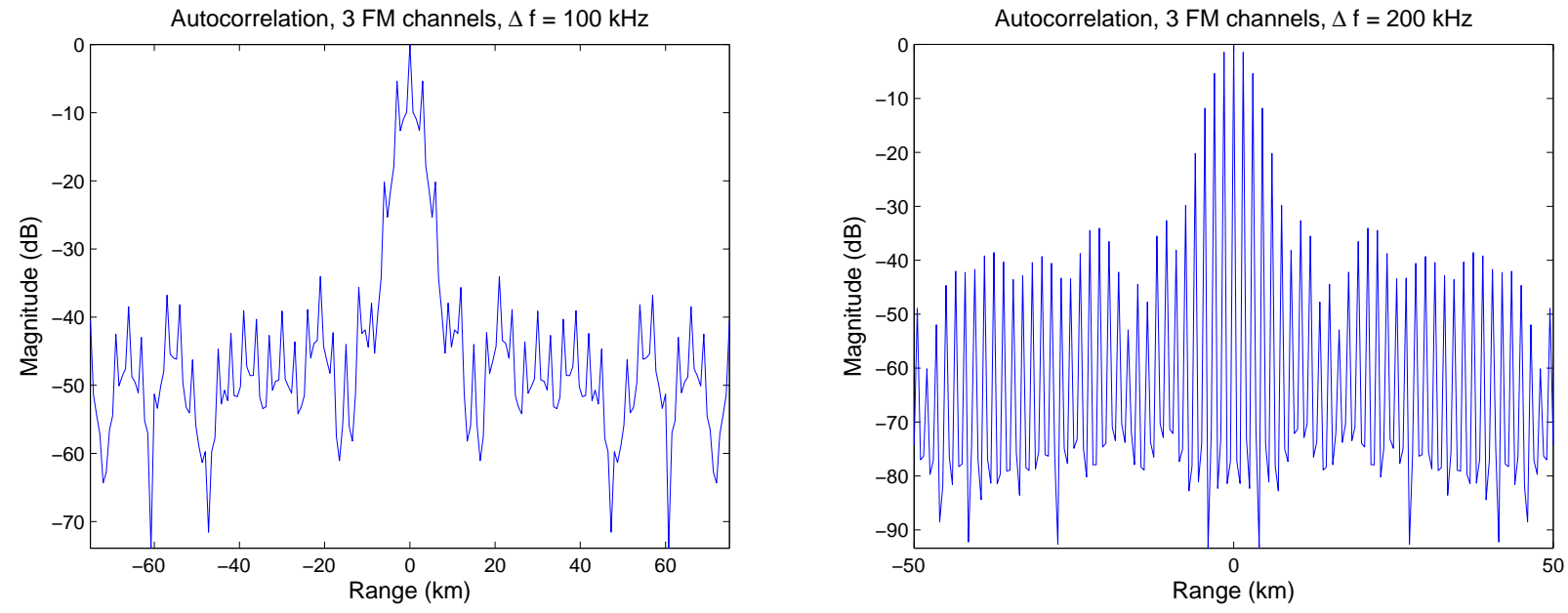

(b) Matched filter results for $M=3 \mathrm{FM}$ channels

(c) Matched filter results for $M=3 \mathrm{FM}$ channels c) with $\Delta f=200 \mathrm{kHz}$ and scenario table 1 .

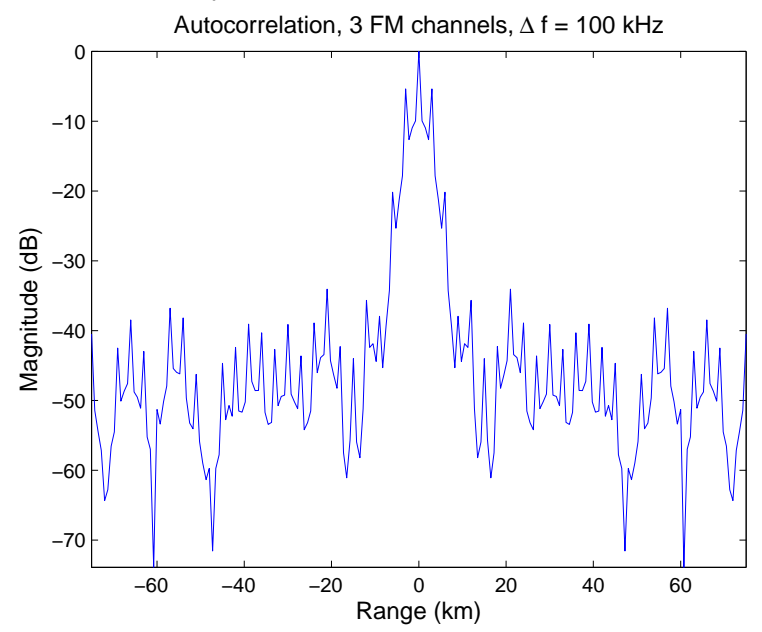

(d) Matched filter results for $M=5$ FM channels with $\Delta f=100 \mathrm{kHz}$ and scenario table 1 .

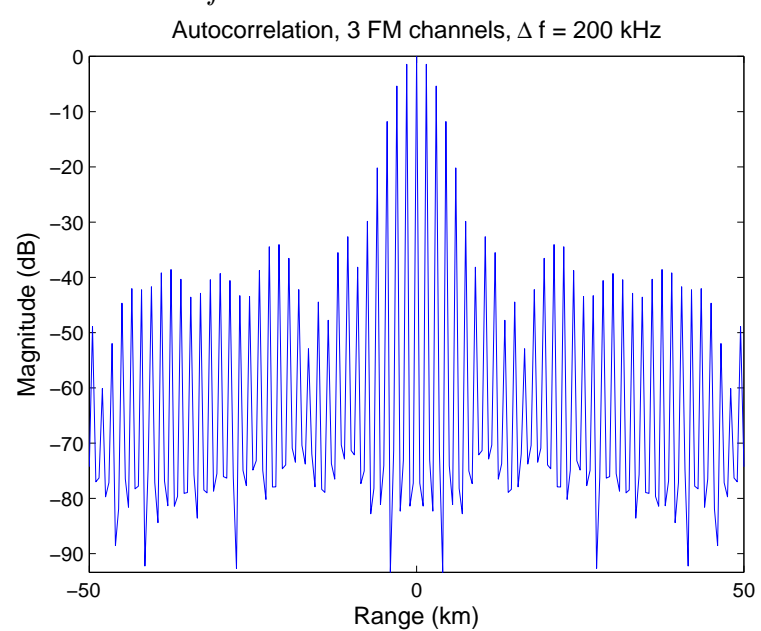

(e) Matched filter results for $M=5 \mathrm{FM}$ channels (e) with $\Delta f=200 k H z$ and scenario table 1 .

Figure 5: Visualization of side-lobe issue in matched filter for multichannel FM signal for 2. 


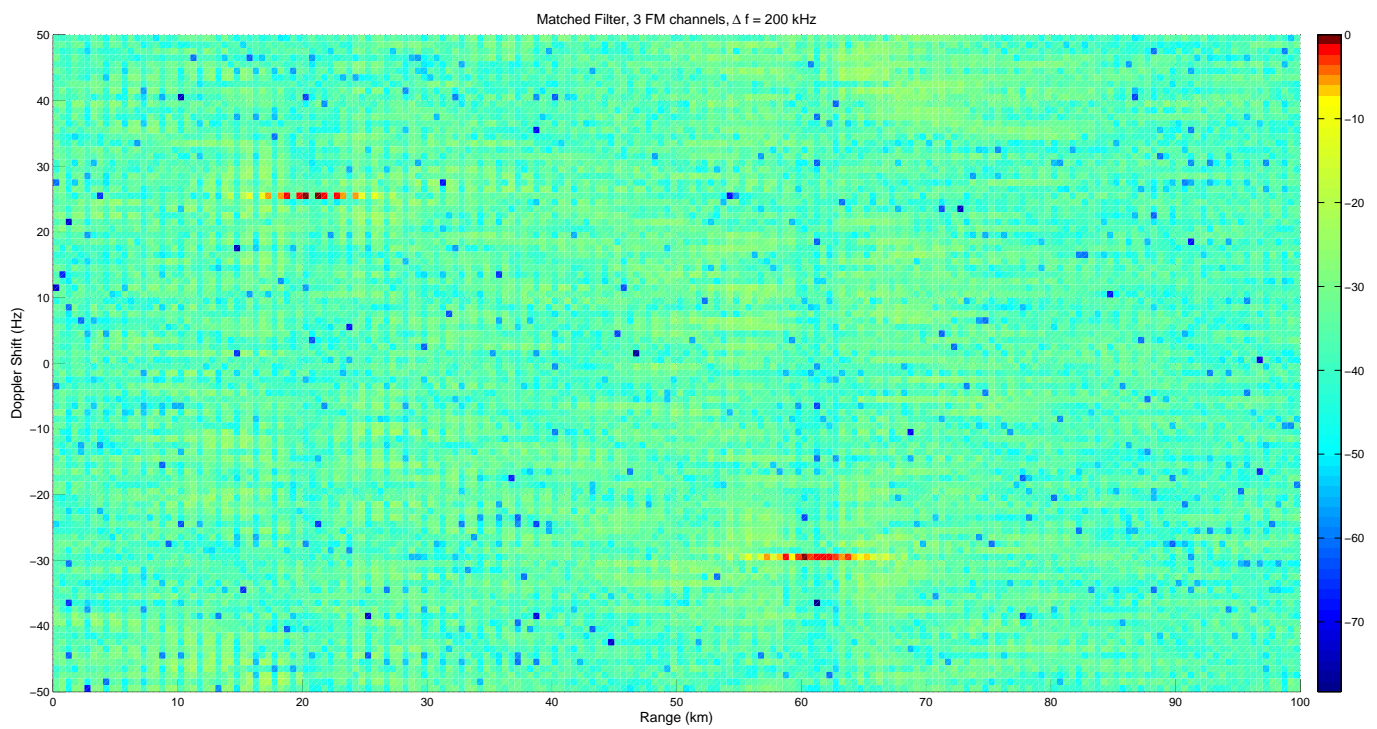

(a) Matched filter results for scenario in Table 3 for $M=3$ channels and $\Delta f=200 \mathrm{kHz}$ without deconvolution.

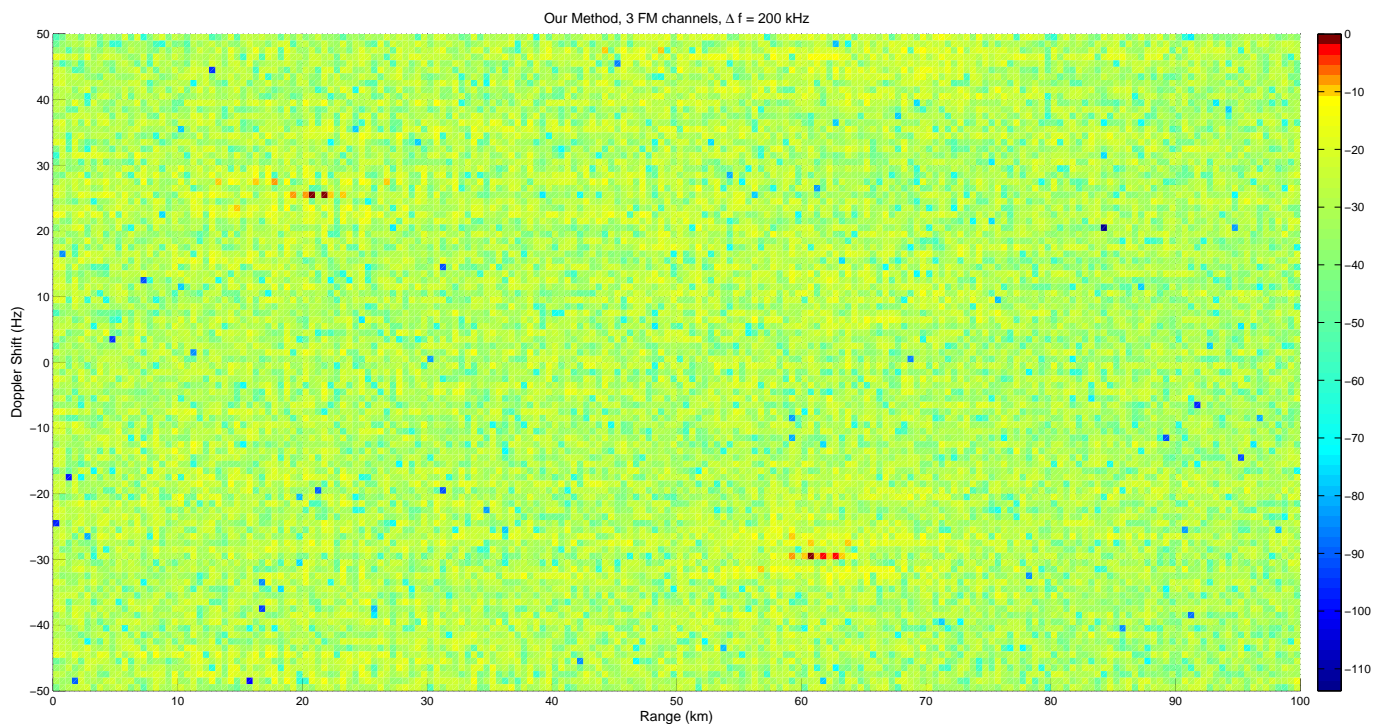

(b) Matched filter results for scenario in Table 3 and for $M=3$ channels and $\Delta f=200 \mathrm{kHz}$ with deconvolution.

Figure 6: Range-Doppler map results for example 3. 


\section{REFERENCES}

[1] Baker, C., Griffiths, H., and Papoutsis, I., "Passive coherent location radar systems. part 2: Waveform properties," IEE Proceedings-Radar, Sonar and Navigation 152(3), 160-168 (2005).

[2] Fuchs, J.-J., "Multipath time-delay detection and estimation," Signal Processing, IEEE Transactions on 47(1), 237-243 (1999).

[3] Griffiths, H. and Baker, C., "Passive coherent location radar systems. part 1: performance prediction," in [Radar, Sonar and Navigation, IEE Proceedings-], 152(3), 153-159, IET (2005).

[4] Di Lallo, A., Farina, A., Fulcoli, R., Genovesi, P., Lalli, R., and Mancinelli, R., "Design, development and test on real data of an fm based prototypical passive radar," in [Radar Conference, 2008. RADAR'08. IEEE], 1-6, IEEE (2008).

[5] Howland, P. E., "Target tracking using television-based bistatic radar," in [Radar, Sonar and Navigation, IEE Proceedings-], 146(3), 166-174, IET (1999).

[6] Tasdelen, A. S. and Koymen, H., "Range resolution improvement in passive coherent location radar systems using multiple fm radio channels," (2006).

[7] Olsen, K. E. and Woodbridge, K., "Performance of a multiband passive bistatic radar processing schemepart i," Aerospace and Electronic Systems Magazine, IEEE 27(10), 16-25 (2012).

[8] Tofighi, M., Kose, K., and Cetin, A., "Denoising using projections onto the epigraph set of convex cost functions," in [IEEE International Conference on Image Processing (ICIP'14).], (Oct 2014).

[9] Censor, Y. and Herman, G. T., "On some optimization techniques in image reconstruction from projections," Applied Numerical Mathematics 3(5), 365-391 (1987).

[10] Chambolle, A., "An algorithm for total variation minimization and applications," J. Math. Imaging Vis. 20, 89-97 (Jan. 2004).

[11] Bregman, L., "Finding the common point of convex sets by the method of successive projection.(russian)," \{USSR\} Dokl. Akad. Nauk SSSR 7(3), $200-217$ (1965).

[12] Cetin, A. E., Bozkurt, A., Gunay, O., Habiboglu, Y. H., Kose, K., Onaran, I., Sevimli, R. A., and Tofighi, M., "Projections onto convex sets (POCS) based optimization by lifting," IEEE GlobalSIP, Austin, Texas, USA (2013).

[13] Combettes, P., "The foundations of set theoretic estimation," Proceedings of the IEEE 81, 182 -208 (February 1993).

[14] Slavakis, K., Theodoridis, S., and Yamada, I., "Online Kernel-Based Classification Using Adaptive Projection Algorithms," IEEE Transactions on Signal Processing 56, 2781-2796 (2008).

[15] Kose, K. and Cetin, A. E., "Low-pass filtering of irregularly sampled signals using a set theoretic framework," IEEE Signal Processing Magazine, 117-121 (2011).

[16] Tofighi, M., Bozkurt, A., Kose, K., and Cetin, A., "Deconvolution using projections onto the epigraph set of a convex cost function," in [Signal Processing and Communications Applications Conference (SIU), 2014 22nd], 1638-1641 (April 2014). 